\title{
Automation in Transfusion Medicine
}

\author{
Uwe Cassens \\ Institut für Transfusionsmedizin, Laboratoriumsmedizin und Medizinische Mikrobiologie, Klinikum Dortmund, Germany
}

The current issue of Transfusion Medicine And HemotherAPY focuses on automation in transfusion medicine. Therefore, several reviews and articles in this issue describe historic and current developments in automation of diagnostics in different fields of transfusion medicine.

In 1901, Karl Landsteiner's elucidation of the ABO blood group system was published and established the basis for numberless discoveries of new human blood group antigens and antibodies during the past hundred years. The discovery and identification of human blood groups represented a milestone for a successful administration of compatible blood transfusions and therefore founded the introduction of immunohematology as new scientific subject. Immunohematology covers the analysis of blood group antigens and antibodies and their interactions in health and disease [1].

Nevertheless, for nearly the whole last century, the manual procedures were the only techniques for determination of blood groups and antibodies in the vast majority of laboratories. For many years, the manually performed tube tests were the only standard in immunohematologic laboratories for all routine and scientific questions. These tube tests were later followed and supplemented by more sensitive and specific manual methods, such as solid phase or gel centrifugation techniques. Only since the past few years, partly or fully automated systems for blood group typing and cross-matching were introduced in immunohematology to a greater extent. Nowadays, an increasing amount of immunohematologic laboratories uses automated systems for blood group typing and cross-matching in addition to the manual work strategies. Depending on the magnitude of blood donation centers and immunohematologic laboratories, a far developed automation in immunohematology is nowadays realized for many fields in daily routine work. In the current issue, the contributions of A. Dada et al. [2] and G. Wittmann et al. [3] reflect on the automation in immunohematology for compati- bility testing and determination of blood groups using different systems of automation.

Another important issue in transfusion medicine is the transmission of infections by transfusions. This problem may still be serious and sometimes causes severe or fatal courses. Although the frequencies of transfusion transmitted infections caused by known pathogens (such as HIV, HBV, HCV) distinctly decreased during the past years, the risks of transmission until now cannot totally be excluded. A high throughput of blood donors to be tested, the necessity to screen all donors for different infections, and always new pathogens require the continuous deployment of fast and reliable methods. For a safe exclusion of infectious diseases, automated serologic techniques as well as automated systems for genetic determinations of viral nucleic acids are established. The review of A. Schuller and W.D. Roth [4] gives a good overview on different automated systems for serological and genetic blood donor testings for infectious diseases.

In addition, the correct quantification and characterization of blood cells in blood donors, blood components and transfused patients is of high relevance. Both the investigation of circulating cells in blood donors according to current guidelines and the quality control of blood products require efficient systems for determination of cell numbers and types. New hematology counters enable the contemporaneous determination of numerous cells and attributes. Therefore, many features of automated systems for diagnostics in hematology are not only relevant for clinicians but are also essential in transfusion medicine. The review of J. Lehner et al. [5] reflects the historic and current developments in automation of hematologic diagnostics that are nowadays supported and performed by modern and sophisticated hematology cell counters.

In summary, the following reviews and articles focus on automated systems ensuring fast and reliable laboratory analyses. All laboratory units in the field of transfusion medicine need

\begin{tabular}{ll}
\hline KARGER & @ 2007 S. Karger GmbH, Freiburg \\
Fax +497614520714 & Accessible online at: \\
$\begin{array}{l}\text { E-mail Information@Karger.de } \\
\text { www.karger.com }\end{array}$ & www.karger.com/tmh
\end{tabular}

PD Dr. med. Uwe Cassens

Institut für Transfusionsmedizin, Laboratoriumsmedizin und Medizinische Mikrobiologie Klinikum Dortmund

Alexanderstraße 30, 44137 Dortmund, Germany

Tel. +49 231 95321-590, Fax -094

E-mail uwe.cassens@klinikumdo.de 
and realize a high extent of automation in order to fulfill the high quantitative and qualitative demands. In the future, the grade of automation in immunohematology, immunogenetics, infectiology and hematology probably will further increase. Innovative laboratories will mainly influence this development as customers and advisers for manufacturers of automated systems.

Nevertheless, even the highest degree of automation will never absolve the scientific and non-scientific staff from final responsibility for accurate work with automated systems and correct interpretation of laboratory results generated by automation.

\section{References}

1 Cruse JM, Lewis RE: Immunohematology; in: Historical Atlas of Immunology. London, Taylor and Francis,-2005, pp 207-223.

2 Dada, Beck D, Schmitz G: Automation and Data Processing in Blood Bank Using the Ortho AutoVue ${ }^{\circledR}$ Innova System. Transfus Med Hemother 2007;34(5): DOI $10.1159 / 000106558$.

3 Wittmann G, Frank J, Schramm W, Spannagl M: Automation and data processing with the Immucor Galileo ${ }^{\circledR}$ system in a university blood bank. Transfus Med Hemother 2007;34(5):DOI 10.1159/000107936.

4 Schuller A, Roth WK: Automation of Blood Donor Testing for Infectious Diseases. Transfus Med Hemother 2007;34(5):DOI 10.1159/000106839.

5 Lehner J, Greve B, Cassens U: Automation in Hematology. Transfus Med Hemother 2007;34(5):DOI 10.1159/000107368. 